\title{
Anabases
}

ANABASES Traditions et réceptions de l'Antiquité

6 | 2007

Varia

\section{Vie et belle santé de la corruption dans la pensée politique de Pascal}

\section{Anne Frostin}

\section{(2) OpenEdition}

1 Journals

Édition électronique

URL : http://journals.openedition.org/anabases/3301

DOI : 10.4000/anabases.3301

ISSN : 2256-9421

Éditeur

E.R.A.S.M.E.

\section{Édition imprimée}

Date de publication : 1 octobre 2007

Pagination : 53-65

ISSN : 1774-4296

\section{Référence électronique}

Anne Frostin, «Vie et belle santé de la corruption dans la pensée politique de Pascal », Anabases [En ligne], 6 | 2007, mis en ligne le 01 janvier 2012, consulté le 20 octobre 2019. URL : http:// journals.openedition.org/anabases/3301 ; DOI : 10.4000/anabases.3301 
Anabases 6 (2007), p. 53-65.

\title{
Vie et belle santé de la corruption dans la pensée politique de Pascal
}

ANNE FROSTIN

\begin{abstract}
D ANS Un FRAGMENT CÉLÈBRE des Pensées, Pascal use d'une image frappante et radicale à propos des traités politiques de Platon et d'Aristote; il nous enjoint de ne pas nous méprendre et de considérer que "... S'ils ont écrit de politique, c'était comme pour régler un hôpital de fous. Et s'ils ont fait semblant d'en parler comme d'une grande chose, c'est qu'ils savaient que les fous à qui ils parlaient pensaient être rois et empereurs. Ils entrent dans leurs principes pour modérer leur folie au moins mal qu'il se peut ${ }^{1}$ ". Il ne s'agit donc pas de guérir mais de donner une allure viable, «modérer leur folie au moins mal qu'il se peut " selon la formule qui clôt le fragment. Cette lecture de Pascal restitue bien le sens de l'énoncé désenchanté que profere l'Athénien dans les Lois : "Assurément les affaires humaines ne valent pas qu'on les prenne au grand sérieux ; cependant nous sommes forcés de les prendre au sérieux et c'est là notre infortune $^{2}$. » Cette assimilation du politique à un désordre généralisé auquel il faut donner une apparence d'ordre et de forme - il s'agit bien de régler - est un motif central de la pensée politique pascalienne. Il nous faut feindre que tout ceci, l'ordonnance du pouvoir comme celui de la société civile, est conséquent et d'importance, feindre que la place de chacun est véritablement fondée. L'insistance de Pascal à souligner ce dérèglement, cette " folie " qui transitent les relations au sein de l'ordre civil est manifeste, ainsi : " La puissance des rois est fondée sur la raison et sur la folie du peuple, et bien plus sur la folie ${ }^{3}$. " Le " bien plus » marque combien il y a plus de déraison que de

1 Les Pensées, fragment 533.L'édition de référence utilisée ici est celle de L. Lafuma : Pascal, Euvres complètes, Paris, Seuil, 1963, indiquant que tous les autres fragments seront tirés de cette édition.

2 Platon, Lois, 803 b, Paris, Les Belles Lettres, 1956, p. 33.

3 Les Pensées, fragment 26.
\end{abstract}


raison, l'aune à laquelle nous allons devoir mesurer est celle de la " folie ». Pascal reprend vigoureusement ce thème usuel, voire éculé de la folie du monde. Les Anciens comme les chrétiens pointaient cette folie profonde qui enveloppe les mouvements des hommes, Cicéron comme Saint Augustin insistent sur leur nombre : "Le monde est

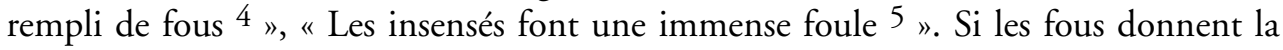
mesure alors « les hommes sont si nécessairement fous que ce serait être fou par un autre tour de folie de n'être pas fou ${ }^{6}$ ».

Il faut donc écrire de la politique comme pour un " hôpital de fous ". Le corps politique est malade, il vit et se nourrit de fictions et d'illusions. Un monde comme à l'envers. S’agit-il alors de déplorer cet état, de soupirer vers ce qui devrait être ou pourrait être ? Tout au contraire reconnaître les nécessités de fait et les impératifs de l'action politique dénote la force de pensée des acteurs obligés de cette " méchante pièce ". "Qui voudrait ne suivre que la raison serait fou prouvé ${ }^{7}$. " Méchante pièce, l'expression doit être prise littéralement car les acteurs - à savoir nous - se définissent par leur seule fureur de domination, leur appétit de pouvoir et de possession. Cette pathétique humaine nous oblige sans rémission à mettre au cœur et comme moteur du dispositif politique, la concupiscence humaine.

La concupiscence est ce qui rend l'individu soumis à la seule loi de son infinie convoitise, de son désir d'avoir et de puissance. Reprenant les préceptes augustiniens, Pascal y voit la marque même de notre nature corrompue et déchue. Corruption qui a pour effet de jeter le soupçon sur la rectitude de tout acte. La concupiscence engendre nécessairement des conduites corrompues puisque ce qui meut chacun est son appétit, son affect et peu le service désintéressé pour le royaume ou l'ordre commun. Chacun est prompt à dissimuler, transiger au nom de sa nécessité personnelle, cela conduit à altérer les mouvements mêmes du corps social. C'est sur le fond de cette nature dévoyée que doit s'ordonner le politique. La corruption et la concupiscence s'exercent avec une telle fureur qu'il semble impossible d'en tirer quelque mouvement rationnel ou sain. Seule la logique des conduites passionnelles conditionne tant la genèse que la pérennité de l'État.

Ce tableau de l'ordre politique en souligne la violence potentielle et la misère. La force de la corruption est telle qu'elle flétrit les conduites, nul juste universel, nulle loi naturelle auxquels on puisse univoquement se rapporter, au contraire tout est sujet à controverse et dispute, aucun principe qui n'ait trouvé sa stricte raison contraire. Le lieu commun de la diversité des lois qui, selon les nations ploient toutes en des sens

4 Cicéron, Lettres familières, IX, 22, 4.

5 Saint Augustin, Contre les académiciens, Paris, Gallimard, 1998, livre I, I, 1, P. 4.

6 Les Pensées, fragment 412.

7 Les Pensées, fragment 44. 
contraires marque de façon irrévocable l'inanité et l'impuissance des entreprises de fondation rationnelle du politique ${ }^{8}$.

De cette loi naturelle, Pascal ne cesse de démontrer qu'elle est vaine, impuissante, indécidable, inconnaissable et toute prétention de recours à elle est source des plus grands maux pour la société civile. Que faut-il donc mettre à la place de l'injustice ? "Que dira-t-on qui soit bon ? De ne point tuer ? non, car les désordres seraient horribles, et les méchants tueraient tous les bons. De tuer ? non, car cela détruit la nature ${ }^{9}$. " Toute tentative de fonder de nouveau est vaine pour autant que c'est tragiquement oublier qu'en cet ordre tout est contestable, rien sur quoi tous puissent s'accorder, au contraire, il n'y a pas pires fauteurs de trouble que ceux qui veulent instaurer un nouvel ordre de justice, les maux en sont encore bien pires, en cela s'exprime d'ailleurs tout le pessimisme pascalien qui nous reconduira à un nécessaire conservatisme.

L'image de "l'hôpital de fous " est donc bien la plus apte à nous donner le juste tableau du politique et destituer celui-ci de toute dignité, il souffrirait d'une sorte de mauvaise santé chronique. La cité est malade, nous retrouvons là un thème récurrent de la pensée politique des Anciens. Cependant le vocabulaire dont use Pascal pour évaluer l'ordre civil mis en œuvre par le politique peut au contraire nous étonner tant par sa santé que par sa force laudative :

On a fondé et tiré de la concupiscence des règles admirables de police, de morale et de justice ${ }^{10}$.

8 Précisons qu'il ne s'agit pas de nier l'existence de la loi naturelle mais nous ne pouvons nous y conformer car notre état de corruption nous en éloigne irrémédiablement. C'est ainsi "que l'éclat de la véritable équité " n’a pas réussi à éclairer les peuples (fragment 60). De même le fragment 86 : «Veri juris. Nous n'en avons plus. Si nous en avions nous ne prendrions pas pour règle de justice de suivre les mœurs de son pays. " L'enjeu ici est le statut de la loi naturelle. Pascal n'en nie pas la réalité ; en revanche il montre qu'elle n'a strictement aucun efficace, puisque nous ne sommes pas à même de la connaître rationnellement et a fortiori de nous y référer. Pascal insiste au contraire sur " la confusion " qui règne à son sujet, donnant lieu à diverses conceptions de l'essence de la justice. Jean Mesnard insiste fortement : "Connaît-il la vraie justice qu'il n'en a pas pour autant la volonté de la réaliser ; et d'ailleurs sa connaissance même est souvent brouillée par l'interférence du péché source perpétuelle de confusion entre l'intérêt et la valeur, entre l'amour de soi et l'amour de Dieu ", J. Mesnard, Les Pensées de Pascal, Paris, SEDES, 1993. Laurent Thiroin voit dans la reconnaissance de cet effacement de la référence à la loi naturelle une preuve du réalisme pascalien (cf. L. THIROIN, Le hasard et les règles : Le modèle du jeu dans la pensée de Pascal, Paris, Vrin, 1991, chapitre IV, p. 61 et suiv.). Sur cette question voir Ch. LaZERri, Force et justice dans la politique de Pascal, Paris, PUF, 1993, p. 201-205. Notamment le chapitre II de la deuxième partie et l'apparat de notes - en particulier 33 et 42 - qui fait état des controverses sur la loi naturelle. 
Les raisons des effets marquent la grandeur de l'homme d'avoir tiré de la concupiscence un si bel ordre 11 .

Grandeur de l'homme dans sa concupiscence même d'avoir su tirer un règlement admirable et en avoir fait un tableau de charité 12 .

Un «si bel ordre " : l'expression est reprise plusieurs fois. Marquons immédiatement la force de ce paradoxe, du désordre procède un ordre, du dérèglement des règles et même le plus admirable des règlements, de la déraison, la folie la plus profonde une raison. Comment devons-nous lire et interpréter cette formule " un si bel ordre " ? Quel statut et quelle place lui accorder ? Trois lectures semblent prédominantes.

S'agit-il d'un un effet rhétorique qui soulignerait la distanciation amère, le désenchantement du propos pascalien ? Les termes ne devant pas être pris littéralement mais comme un procédé ironique qui permettrait à Pascal, certes, de marquer la pertinence de cet ordre institué, établi mais aussi de dénoncer sa fausseté et son inconsistance essentielle au regard du seul véritable ordre à savoir celui de la charité. Somme toute, ce à quoi l'on parvient n'est qu'un dérisoire " tableau de charité " qui procède du seul jeu des concupiscences, l'admiration devient sujette à caution car en son fond elle nous renvoie à notre corruption.

Cette manœuvre proprement pascalienne - à savoir cet usage de l'ironie - est à l'œuvre sous des modalités diverses, nullement réductibles à des antiphrases ou à la simple raillerie - fondamentalement, elle s'avère en dernier ressort, un jeu qui met à jour ce qui est dissimulé et démasque le double sens des rapports ${ }^{13}$. Il est intéressant de souligner que Jean Mesnard prend ce fameux fragment 533 (l'hôpital de fous) comme une des figures exemplaires de l'ironie puisqu'il mettrait en ouvre une feinte adopter le point de vue de l'interlocuteur, en l'occurrence ces fous qui pensent être rois et empereurs - au service d'un renversement de ce même point de vue. S'agirait-il du même procédé pour les fragments relatifs au si bel ordre ? Il prêterait fondamentalement et tragiquement à rire.

Au contraire, ne nous faut-il pas prendre acte de ce "bel ordre ", prendre acte de ces " règles admirables ", de cette régulation, de cet ordre dont on doit reconnaître la pertinence et qui ouvre bien à un affinement, à un perfectionnement tels que l'État participe d'une assomption de l'intérêt privé dans l'intérêt collectif, assomption telle qu'on peut en attendre le meilleur? On accorde alors à l'adjectif " admirable » une réelle portée et on en tire la preuve que seule la soumission à ces lois civiles essentielles à la

11 Les Pensées, fragment 106.

12 Les Pensées, fragment 118.

13 J. Mesnard, Les Pensées de Pascal, III partie, chapitre I, p. 286 : «Le fragment sur Platon et Aristote enferme d'ailleurs tous les éléments d'une définition de l'ironie ». L. THIROUIN, "Imprudence et impudence, le dispositif ironique dans les Provinciales", Courrier du centre international Blaise Pascal, 18 (1996). 
conservation du tout donne un fondement consistant et marque la grandeur de ce " tableau de charité ». Le vocable " admirable " reçoit alors une véritable consistance, comme le remarque G. Ferreyrolles 14 il est utilisé par Pascal dans le fragment 211 comme dans le fragment $454{ }^{15}$ où il désigne les commandements reçus de Dieu par le peuple juif.

Ou encore s'agit-il d'une stricte leçon de réalisme qui ne débouche ni sur la fausseté constitutive dénoncée ni sur un perfectionnement possible mais sur l'efficace de la loi, du jeu des règles. On lirait alors le terme « admirable » comme pur constat d'une efficace dont la portée réside essentiellement dans sa capacité inouïe à instituer un ordre, un «si bel ordre » et en ce sens sa force résiderait justement à effacer l'arbitraire, parce que l'usage est souverainement impérieux alors la règle vaut forcément, admirable est son règlement. Il n'y a pas d'autre ordre plus consistant et plus réel que cette apparence d'ordre. Le tableau de charité seul nous rappellerait notre portée pour autant qu'on s'y référerait.

Le nœud qui unit contradictoirement l'affirmation que l'on ne peut écrire de politique que pour " régler un hôpital de fous » et la reconnaissance réelle (j’entends effective) de la valeur de ces mêmes règles comme " admirables " permet de manifester la relation double à la règle corrompue en son fond et d'ouvrir à l'originalité de l'analyse effectuée par Pascal. Le regard éclairé sur l'ordre civil doit savoir reconnaitre la santé de ce jeu de trompe-l'œil en même temps qu'il ne se laisse pas abuser par lui, ce qui veut dire selon le vocabulaire de Pascal, en comprendre la portée.

Nous comprenons bien, alors, que ce qui se joue est le sens de cette duplicité constitutive du politique. La lancinante accusation de machiavélisme de la politique pascalienne - de combien il en relève, jusqu'où côtoie-t-il Machiavel ? - se joue bien dans cette ambiguïté à laquelle nous reconduit l'efficace de l'économie de la concupiscence et de la corruption.

Économie vraiment " admirable » car elle assure un ordre que l'on peut considérer comme aussi performant que celui qu’aurait pu produire une belle déduction du droit naturel - ainsi le fragment 977 : « Les choses du monde les plus déraisonnables deviennent les plus raisonnables à cause du dérèglement des hommes ». Nous devrons relever ce vocabulaire qui marque cette santé de l'ordre qui émane de notre état corrompu. Une compréhension exclusivement tragique de cet artifice de la loi comme si celle-ci n'avait pour seule fonction que de nous rappeler combien nous sommes méprisables de n'obéir qu'à des simulacres, des grimaces et des parodies de justice

14 G. Ferreyrolles, Pascal et la raison du politique, Paris, PUf, 1984. Chapitre IV, note 3, p. 199. C'est là un argument essentiel pour une interprétation qui légitime pleinement l'usage du vocable « admirable».

" Je considère cette loi qu'ils se vantent de tenir de Dieu et je la trouve admirable. C'est la première loi de toutes et de telle sorte qu'avant même que le mot loi fût en usage parmi les Grecs, il y avait près de mille ans qu'ils l'avaient reçue et observée sans interruption... », fragment 454 . 
mésestime la puissance d'organisation du jeu politique et le sens du jeu, ce n'est peutêtre que du seul point de vue de la charité que le jeu est si indigne. Dès lors, prenons acte de ce si bel ordre et considérons jusqu'où il peut-être pris comme marque de santé. Jusqu'où le coup de force du règlement peut-il être affirmé comme signe de la performance de la règle?

Toute approche seulement ironique ou dénonçant l'indignité constitutive du politique n'est-elle pas contredite par ces propos de Pascal rapportés par Pierre Nicole à propos de l'instruction d'un prince : " Il n'y avait rien à quoi il désirait plus de contribuer, s'il y était engagé et qu'il sacrifierait volontiers sa vie pour une chose si importante ${ }^{16}$."

Comment tenir sans contradiction ces trois énoncés successifs sur la règle en politique : "l'hôpital de fous ", "son si bel ordre ", " cette chose si importante " ? S'ils disent, chacun, un sens du politique dont chaque interprète peut légitimement s'autoriser, l'ordre du politique semble concentrer en lui la plus grande puissance d'amplitude de la règle, jusqu'à ériger un ordre performant d'apparences. Si bien que ce jeu artificiel recèle une étonnante fécondité. Nous devons exhiber la force de composition qui définit la règle politique et nous interroger sur le sens de ces doubles rapports qu'elle ne cesse de mettre en œuvre.

Lorsque Platon dénonce l'état malsain de "la cité phlegmoneuse 17 ", il oppose à cela le bon geste politique qui devrait être semblable à un geste thérapeutique, celui du médecin hippocratique. Il fustige ces politiques, tels des cuisiniers coupables de gaver la cité jusqu'à l'embonpoint, de lui donner « le mal de gloutonnerie " la menant ainsi à « l'enflure et à la suppuration 18 ». Les Périclès, Thémistocle, Cimon, "ils ont traité $<$ les citoyens> en les régalant magnifiquement selon leurs désirs 19 ». La définition du statut de "Grand » que pose Pascal se rapproche à bien des égards de ces figures, dans la mesure où il s'agit là aussi d'une économie des désirs. Ces grands dominés par leur désir de dominer dépendent de leurs propres sujets puisqu'ils se font les serviteurs de leurs désirs.

Qu'est-ce, à votre avis, d'être grand seigneur ? C'est être maittre de plusieurs objets de la concupiscence des hommes, et ainsi pouvoir satisfaire aux besoins et désirs de plusieurs... c'est la concupiscence qui les attache à vous... Vous êtes donc proprement un

Les Pensées, fragment 1004.

Platon, La République, 372 e. Selon la traduction de H. Joly qui donne une traduction littérale : H. JolY, Le renversement platonicien. Logos, Epistémè, Polis, Paris, Vrin, 1974, Chapitre III, IIIe partie, p. 240, note 49, ou "cité gonflée d'humeurs" selon la traduction de G. Leroux (Platon, La République, Paris, Flammarion, « GF », 2002.

8 Platon, Gorgias, 518c-519a. Selon la traduction de H. Joly in Le renversement platonicien [n. 17], ou "Cité toute enflée de pus" selon la traduction de M. Canto (Platon, Gorgias, Paris, Flammarion, «GF », 1987.

Platon, Gorgias, 518e, trad. par H. Joly in Le renversement platonicien, [n. 17], p. 240. 
roi de concupiscence... C'est la concupiscence qui fait leur force, c'est-à-dire la possession des choses que la cupidité des hommes désirent ${ }^{20}$.

Rois, riches, capitaines sont ces " grands de chair ». Or l'ordre de la chair nous enferme dans cette "libido dominandi " qui nous meut, " tous les hommes voulant dominer et tous ne le pouvant pas, mais quelques-uns le pouvant 21 ". Seul le recours à la force peut mettre un terme à cette guerre. Le pouvoir s'explique fondamentalement en termes de force : "Droit d'épée " dit de façon laconique Pascal. Cependant cette force brute est insupportable, il faut donc qu'elle soit recouverte, transfigurée en apparence de justice : "Il ne faut pas qu'il sente la vérité de l'usurpation, elle a été introduite autrefois sans raison, elle est devenue raisonnable. Il faut la faire regarder comme authentique, éternelle et en cacher le commencement, si on veut qu'elle ne prenne bientôt fin ${ }^{22}$."

C'est l'imagination qui œuvre à cette conversion maligne de force en justice et en droit grâce à un jeu symbolique de signes, de noms. Cette série de « déplacements " par imagination promeut des figures fictives de respect, de terreur, de fascination. Pour cela Pascal s'attarde à décrire les signes vestimentaires par lesquels les différents corps se distinguent et se "sacralisent ": "Magistrature fourrée dans les robes rouges et les hermines, soutanes et mules des docteurs, bonnets carrés des médecins, les gardes, les balafrés des Rois 23 ", mais aussi tous ces signes extérieurs de puissance et de richesse : " homme vêtu de brocatelle, escorte de sept à huit laquais ${ }^{24}$..."

La mystification est la condition du règne. C'est justement dans le décryptage de cet arsenal de signes et dans la mise à nu des différents ressorts et rouages des dispositifs du pouvoir que va s'exercer l'analyse et l'originalité de la pensée pascalienne.

Ils se trompent ceux qui veulent guérir le politique en le soumettant à des principes qui ne sont pas de son ordre Pascal assume cette autonomie du politique, seule condition par laquelle on puisse coïncider avec ses impératifs, ses contingences et ce, sans se leurrer sur l'ordre de ses raisons. Ici, pour nous, il ne s'agit que de force, de coutume, de dissimulation. La distance est si grande entre le modèle de la république chrétienne qui se suffit de "deux lois " et la pléthore de nos lois ${ }^{25}$. La concision du

Trois discours sur la condition des grands. Troisième discours, Paris, Seuil, 1963, p. 367.

Les Pensées, fragment 828. Marquons bien cette progression de " tous » à " quelques-uns ", il s'agit de partis, de factions comme "France des gentilshommes ", "Suisse des roturiers", " la pluralité » ou dans un vocabulaire actuel : la majorité. Il ne s'agit jamais d'entente concertée de chacun s'en remettant par la voie d'un contrat à un autre; ce sont de purs rapports de domination sous les plus forts.

Les Pensées, fragment 60.

Les Pensées, fragment 44.

Les Pensées, fragment 89.

Les Pensées, fragment 376 : "Deux lois suffisent pour régler toute la République chrétienne, mieux que toutes les lois politiques. " 
propos renforce la perfection de ce minimalisme juridique, cet écart accuse d'ailleurs l'inadéquation de la loi de l'une pour les lois de l'autre. Pascal ne ménage strictement aucune place à l'idéal du Prince chrétien, nulle issue à attendre du côté de la religion ou du théologique, il dénonce d'ailleurs ces dévots "qui ont plus de zèle que de science 26 " qui croient pouvoir bouleverser la société et accuser le pouvoir au nom de lumières supérieures 27 . Parfaite leçon de réalisme politique.

L’ordre du politique tel que Pascal le détermine se développe sous le signe des apparences et de la tromperie ou autre piperie selon le vocabulaire pascalien, toute possibilité d'une fondation ou d'une déduction rationnelle d'une pratique politique étant niée, on ne trouvera aucune interrogation sur les différentes formes de gouvernement ni même de tentative de légitimation du pouvoir ou de théorie politique. Pascal part de la société telle qu'elle est constituée, or c'est bien à un seul jeu d'images déformantes et déformées du pouvoir qui sont autant de dissimulation et de cache misère d'une usurpation initiale que nous sommes ramenés.

En quoi tout ceci peut-il recevoir l'épithète de "si bel ordre " ? La liasse V, intitulée "Raison des effets " (fragments 80 à 104) qui dissèque et exhibe les mécanismes sociaux nous permet de le justifier. L'organisation sociale est bien appropriée sous ses absurdités manifestes. Il s'agit d'un système fondé sur l'apparence, les signes du pouvoir doivent être immédiatement reconnaissables et visibles, d'une lisibilité frappante. Pascal différencie deux modalités ou selon ses propres termes deux qualités : les qualités spirituelles et les qualités palpables, or il est manifeste que des unes et des autres on ne se sert pas également. Le fragment 85 des Pensées est sans détour : "...mais comme la force ne se laisse pas manier comme on veut parce que c'est une qualité palpable, au lieu que la justice est une qualité spirituelle dont on dispose comme on veut ". La

26

Les Pensées, fragment 90.

La dénonciation virulente par les dévots de la politique mondaine qu'incarne alors Richelieu échoue à se convertir dans une théorie politique définie. Face aux nécessités et aux contingences temporelles ces traités restent bien silencieux. L'art politique chrétien demande " une sagesse au dessus de l'esprit et la capacité d'un homme ", il semble en tout état de cause bien impuissant face à ce que tous les dévots appellent d'une seule voix « la raison d'enfer ». Raison d'enfer, les écrits dévots rappellent au roi qu'il n'est que le vicaire de Dieu, énoncé récurrent des pamphlets dévots contre le pouvoir de l'État profane : "Tous les monarques de la terre, n'étant que les Vices-rois de ce grand Roi, gouverneront heureusement, si longtemps qu'ils prendront ses avis et suivront ses ordonnances. " Le testament politique de Richelieu demandera aux sujets, à travers une maxime qui fera horreur aux dévots, de faire régner la raison - d'État - de telle sorte que ceux qui sont sous son autorité « la révèrent et la suivent religieusement ». Pernicieux raisonnement, aux yeux des dévots, qui s’appuie sur l'interprétation la plus sophistique du mot du Christ : « Mon royaume n'est pas de ce monde ", H. MUGNIER, La véritable politique du Prince chrétien à la confusion des sages du monde et pour la condamnation des politiques $d u$ siècle, Paris, S. Piquet, 1647. Sur cette question, cf. E. ThuAU, Raison d'État et pensée politique à l'époque de Richelieu, Paris, Armand Colin, 1966 ; réédition Paris, Albin Michel, 2000. 
distinction peut apparaître bien faible conceptuellement voire caricaturale mais ce serait se méprendre. En effet, puisqu'il s'agit de ces rapports entre force et justice, reléguer la justice du côté du spirituel c'est simplement marquer qu'elle relève de la pure réflexion qui nécessite le langage, les définitions, les nominations, les effets de sens. Or qu'est ce qui peut être plus maniable que le langage ordinaire ? C'est le lieu par excellence de l'interprétation, de l'équivoque, de la contradiction, de la controverse, en un mot de la dispute en tous les sens du terme. Tout peut apparaître ici éminemment contestable, ce qui offre les possibilités des plus grandes de falsifications ${ }^{28}$. Dans l'ordre des valeurs, des choix, des raisonnements qui ne se cantonnent pas à l'ordre géométrique, l'incertitude et la contestation règnent. Travestir les mots, se faire aveugle au sens figuré et au sens littéral, permet si aisément de tromper et d'introduire le mensonge par les artifices de la langue. Cette dimension est essentielle car elle justifie le privilège des " qualités palpables " qu'offre la force.

L'une des qualités est sujette à dispute, l'autre non. Dès lors que la loi naturelle est comme effacée, que nous ne connaissons pas la justice alors il faut se régler sur autre chose. Est-il nécessairement injuste de substituer la force au juste ? Le fragment 744 nous éclaire sur l'importance de la règle : "Lorsqu'on ne sait pas la vérité d'une chose, il est bon qu'il y ait une erreur commune qui fixe l'esprit des hommes... car la maladie principale de l'homme est la curiosité inquiète des choses qu'il ne peut savoir, il ne lui est pas si mauvais d'être dans l'erreur que dans cette curiosité inutile ". En l'absence de toute définition rigoureuse du juste plutôt que de verser de contestation en contestation au nom de sa fondation, il faut s'en remettre à la puissance et l'autorité de la force comme capacité à régler. Or il est avéré que la raison du plus fort - force de la pluralité, force du roi, force des institutions - est efficace par sa lisibilité et permet un monde bien ordonné. Certes les qualités qu'elle met en avant sont des plus superficielles - au sens littéral du terme : "C'est montrer par ses cheveux... par son rabat, le fil, le passement 29 ... ", mais parfaitement adéquates à leur office. Signes indubitables par lesquels nous plions et consentons à ces grandeurs d'établissement. Tout au contraire nous faut-il faire l'éloge des apparences, il s'agit non de faux-semblants mais de la force du " dehors " par lequel les acteurs de la scène sociale se reconnaissent à leurs marques visibles ${ }^{30}$. La force de la

28 Nous pourrions puiser dans Les Provinciales des exemples remarquables de l'art des jésuites à user et jouer des équivoques, aller contre le sens commun, favoriser les renversements de sens. Ils abusent des définitions. Seconde lettre des Provinciales, voir l'analyse de l'usage du terme "suffisant " par les jésuites et les dominicains contre le sens commun. Voir également la Sixième lettre des Provinciales et l'introduction d'ambiguïtés dans le terme univoque de superflu. Voir notamment Pierre Force, Le Problème herméneutique chez Pascal, Paris, Vrin, 1989, chapitre III.

Les Pensées, fragment 95. Pascal récuse que tout cela ne soit que "simple superficie ». Les apparences sont effectives, rien de moins superficiel que cette superficie.

Laurent Thirouin a une belle formule : "Tout ce qui compte doit paraître et seul ce qui paraît peut compter ", "Pascal et le savoir vivre", Actes du colloque de l'université de Tokyo, 
loi tient dans sa capacité à être reçue ; là est sa seule réalité. Nous sommes passés de la force brutale aux signes de la force.

Il faut donc considérer sous un tout autre point de vue ce tableau misérable que l'on donnait du politique, les opinions que l'on dénonçait comme vaines deviennent saines sous un autre point de vue : "Elles <les guerres civiles> sont sûres si on veut récompenser les mérites, car tous diront qu'ils méritent. Le mal à craindre d'un sot qui succède par droit de naissance n'est ni si grand, ni si sûr ${ }^{31}$. ". La liasse $\mathrm{V}$, « Raison des effets ", expose clairement cette dynamique.

Citons l'un des plus célèbres fragments ${ }^{32}$. Le peuple honore les personnes de grande naissance, or rappelons nous ce que vaut la naissance des grands : "Que la noblesse est un grand avantage qui dès 18 ans met un homme en passe, connu et respecté comme un autre pourrait avoir mérité à 50 ans. C'est trente ans gagnés sans peine ${ }^{33}$. " Le peuple croit réellement à cette grandeur.

Les demi-habiles (les réformateurs, les contestataires) vont dénoncer la supercherie, ramener la grandeur à sa réalité : effet du hasard et revendiquer une authentique grandeur, une fondation de la grandeur selon le juste, en conséquence, il ne faut donc pas honorer les grands. Les habiles, eux, renversent ce nouveau point de vue pour assurer qu'il faut se courber devant les grands, non pas pour les raisons du peuple mais par une " pensée de derrière " qui sait rapporter l'effet (on se courbe devant un grand) à sa raison : la nécessité de l'obéissance à des grandeurs qui ne sont que d'établissement et non à des grandeurs naturelles.

«La raison des effets » développe l'ambivalence et la contrariété constitutives sous lesquelles se manifestent les relations au sein de l'ordre politique. Sous un certain rapport seulement les points de vue consécutifs des différents acteurs du jeu politique s'avèrent justes mais ils sont essentiellement incomplets car considérés sous un autre rapport ils sont inadéquats. Tout l'art consiste à savoir démultiplier les points de vue. Cette capacité à saisir et développer ces raisons concurrentes qui engendrent de véritables antinomies donne lieu à des séries de renversements continuels du pour au contre selon la concision de l'expression employée par Pascal au fragment 90 des Pensées.

Par cette " pensée de derrière " nous consentons et comprenons cet ordre dont on reconnaît le désordre. L’ordre sur fond de désordre est le mieux réglé. L'effet apparemment aberrant trouve ses raisons et devient profondément raisonnable ou justifié, il ne s'agit pas de s'en gausser. Ce dédoublement de pensée permet alors une relecture des

27-29 septembre 1988. L'analyse de Gérard Ferreyrolles dans le chapitre "La concupiscence collective" de son ouvrage Pascal et la raison du politique [n. 14], paraît ici incontournable. On pourrait en multiplier les citations, telles que celle-ci : "Comme de l'amour propre surgit la sociabilité, on voit la force enfanter la douceur ", p. 145-146.

Les Pensées, fragment 94.

32 Les Pensées, fragment 90.

33 Les Pensées, fragment 104. 
signes de la force, d'en comprendre la portée. Dans le Premier discours sur la condition des grands, Pascal insiste sur les raisons de cet ordre artificiel de la loi, les législateurs ont eu de "bonnes raisons " mais, précise-t-il, « aucune n'est prise d'un droit naturel ».

Cet ordre aussi superficiel qu'il est artificiel et donc efficace va accoucher de sa figure accomplie : l'honnête homme. L'honnête homme comprend que l'on ne peut réaliser ses propres bonheurs qu'en cédant une place aux autres moi. Un intérêt commun s'édifie sur le jeu des égoïsmes, les mœurs se polissent, le désir d'être estimé nous conduit à vouloir bien faire. Le voilà le bel ordre régulant la scène sociale sur laquelle tous paraissent. Chacun travaille à paraitre et ce faisant s'affine, se police. La règle permet au jeu de se dérouler, chacun tenant sa place. Ces lois arbitraires en leur fond assurent de façon tout à fait acceptable, voire " admirable » leur office.

Faut-il alors conserver le vocabulaire dans lequel nous avons défini les termes de l'ordre politique ? Devons-nous encore nous inscrire dans cette ligne de partage entre le superficiel, l'artificiel et le réellement fondé ?

Or Pascal ne cesse d'insister : en cet ordre de la chair, du politique, on ne sait ce qu'est la loi naturelle, il nous est impossible de discerner ce qui est juste de ce qui est injuste selon les critères de l'authentique justice. Nous disposons des prescriptions de la coutume, de nos lois qui assignent des normes qui nous accoutument à consentir. Les principes sont arbitraires certes mais ils ne sont pas sujets à confusion ou à dispute. Pour comprendre cette règle fondamentale de la scène et du jeu politique il faut revenir à la relation avec le langage.

Dans l'Esprit de géométrie, Pascal montre comment la raison humaine est dans l'incapacité de tout définir et se suffit pour des termes primitifs d'un accord entre les hommes, l'usage fondant la signification ${ }^{34}$. Dans l'ordre du politique, l'accord de convention nous est devenu quasi naturel, dans l'ordre de la langue l'expérience montre une parfaite régularité dans l'usage des mots et des idées, des signes. Cela relève d'une disposition arbitraire mais qui est devenue une quasi nature remarquablement performante : «Nous supposons que tous les (mots) conçoivent de même sorte. Mais nous le supposons bien gratuitement car nous n'en avons aucune preuve... De cette conformité d'application on tire une puissante conjecture d'une conformité d'idée ${ }^{35}$."

Cette citation semble affaiblir la référence à l'accord de convention, mais il n'en est rien, Pascal insiste sur cette dimension d'usage dans le langage, l'usage est un fait de coutume, il est donc arbitraire mais en même temps remarquablement performant, constitutif et souverain. De la même façon dans l'ordre social ce sont les usages qui fondent des accords qui semblent justes et fondés. Il semble toujours injuste de vouloir

34 «Ce n'est pas que tous les hommes aient la même idée de l'essence des choses que je dis qu'il est impossible et inutile de définir ", PASCAL, De l'esprit géométrique, Euvres complètes, éd. citée, p. 350.

Les Pensées, fragment 109. 
menacer et brouiller ces usages ancrés. C'est pourquoi Pascal revient toujours à l'injustice de la fronde, la sottise de ceux qui refusent de rendre "les cérémonies extérieures ", or c'est sur ces postures que se construisent les rapports sociaux, de la même façon, il ne cesse de dénoncer les manœuvres jésuites pour tordre l'usage des termes. Revenons sur l'usage du terme "suffisant " :

Tout ce qu'il y a de personnes au monde entendent le mot de suffisant en un même sens... Toutes les femmes, qui font la moitié du monde, tous les magistrats, tous les gens de palais, les marchands, les artisans, tout le peuple; enfin toutes sortes d'hommes, excepté les Dominicains, entendent par le mot de suffisant ce qui enferme le nécessaire ${ }^{36}$.

Pascal marque conjointement la force de l'usage et sa fragilité, or de la même façon que l'ordre social est infondé pour autant qu'il aurait pu être autre, de la même façon l'ordre de la langue commune est conventionnel, mais les deux sont devenus " nature ", en terme politique le fait s'est converti en droit. Est-il pertinent de continuer à brandir le terme d'arbitraire si force et justice loin d'être opposés sont devenus synonymes ? Il faut aussi écouter l'énoncé maintes fois tourné et retourné par Pascal : ce qui est fort est juste. Il n'est pas nécessaire de connaître le juste pour établir un gouvernement juste.

Mais on pourrait nous opposer le sens de ce vocable : « juste ». À quoi nous référons-nous? Il faut revenir à l'Esprit géométrique : "Ce n'est pas la nature de ces choses que je dis qui est connue de tous : ce n'est que le rapport entre le nom et la chose... car les définitions ne sont faites que pour désigner les choses que l'on nomme, et non pas pour en montrer la nature... ». Il est tentant alors d'exacerber cette force de l'usage qui permet de poser des termes, notions et rapports autour desquels les consensus du sens s'effectuent.

Ce que Pascal ne cesse de rappeler en multipliant les énoncés sur la coutume, la croyance, la machine : "Car il ne faut pas se méconnaitre, nous sommes automate autant qu'esprit... Les preuves ne convainquent que l'esprit, la coutume fait nos preuves les plus fortes et les plus crues. Elle incline l'automate qui entraîne l'esprit sans qu'il y pense. Il faut acquérir une créance plus facile qui est celle de l'habitude qui sans violence, sans art, sans argument nous fait croire les choses et incline toutes nos puissances à cette croyance $37 . . . »$ Le juste auquel nous nous référons n'a pas besoin d'être défini définitivement et absolument, cela donne lieu aux illusions des définitions essentielles, définitions de chose qui ouvrent la porte aux combats, aux pires positions dogmatiques, définitions raides et inflexibles aveugles à leurs faiblesses et leurs manquements. C'est bien pour cela que les appels aux définitions essentielles à propos de la justice sont source de controverses, de polémiques et de combats et l'affaiblissent bien plus qu'elles ne la fortifient. 
Paradoxalement la force peut apparaître bien plus flexible quoique moins maniable puisqu'elle ne prétend aucunement à la fondation. Nous sommes dans un univers d'artifice. Dès lors il ne faut succomber ni aux jeux des apparences ni s'y soustraire sinon on fait preuve soit de superbe et d'orgueil, soit de naïveté si l'on croit à la consistance des lois et rites. Pour cela nous comprenons l'usage du terme de "schizophrénie sociale " employé par Laurent Thirouin 38 et qui rend compte du dédoublement constant que suppose la pensée de derrière. Cette pensée secrète accuse intérieurement mais n'a pas vocation à se manifester. Elle doit constamment occuper l'esprit des « grands ». La folie est d'oublier que cette grandeur n'est que d'emprunt, de convention. Impitoyablement la pensée de derrière reconduit aux raisons de la déraison, à la dérision des rôles.

Mais justement la force de Pascal est de ne renoncer à aucun des points de vue et de soumettre à la différence des perspectives, les règles. La règle pascalienne est d'échapper à toute position univoque; l'effort consiste à ne jamais se limiter à la seule validité d'une assertion afin d'être capable de penser la pertinence de la position adverse et de ne pas succomber à l'idolâtrie d'un point de vue fixe ni tomber dans la situation d'égalité des positions. Cela nous donne une puissance de vue qui permet de saisir la complexité des relations. Le mot d'ordre devient : faire varier les points de vue, allier les contraires afin de traquer toute position exclusive. Pour cela la tentation que nous aurions d'enfermer le politique dans une résolution est contredite par la loi d'ambivalence des perspectives. Nous aurions tous les éléments pour établir un nominalisme juridique ou pour établir un réalisme politique qui conduit inéluctablement à rapprocher Machiavel et Pascal mais la valeur varie selon le point où l'on se situe, une même notion se déterminera donc différemment selon sa place.

"Un si bel ordre », «l'ordre admirable », nous recherchons la portée de ces termes, la valeur de ces épithètes. Or il nous faut reconnaître que ces adjectifs relèvent d'un vocabulaire esthétique, c'est effectivement de l'ordre du tableau, de la scène de théâtre, $\mathrm{du}$ jeu. Ce n'est qu'une figuration, une image. La folie consiste très justement à oublier que tout cela ne vaut que comme artifice : "Mais dans le fond, ce vilain fond de l'homme, ce figmentum malum n'est que couvert. Il n'est pas ôté ». Seul le chrétien parfait connaît l'amplitude de vue et conçoit la portée de la règle ; il comprend la fécondité de cette folie qui est d'obéir à des lois arbitraires par esprit de pénitence ${ }^{39}$, ce point de vue supérieur donne consistance à la folie qui devient éminemment justifiée. L'ambivalence des points de vue est maintenue.

$$
\begin{aligned}
& \text { Anne FrosTiN } \\
& \hline \text { 33, avenue Wilson } \\
& \text { 56400 Auray } \\
& \text { anne.frostin@wanadoo.fr }
\end{aligned}
$$

38 L. Thiroin, "Pascal et le savoir vivre" [n. 30].

39 Les Pensées, fragment 14. On obéit aux folies non parce qu'on les respecte, mais sur "l'ordre de Dieu qui pour la punition des hommes les a asservis à ces folies ». 